\title{
T-S Fuzzy-Based Optimal Control for Minimally Invasive Robotic Surgery with Input Saturation
}

\author{
Faguang Wang $\mathbb{D},{ }^{1,2}$ Hongmei Wang $\mathbb{D}^{1},{ }^{1}$ Yong Zhang $\mathbb{D}^{1},{ }^{\text {and Xijin Guo }}{ }^{1}$ \\ ${ }^{1}$ School of Information and Control Engineering, China University of Mining and Technology, No. 1 Daxue Road, \\ Xuzhou 221116, China \\ ${ }^{2}$ Collaborative Innovation Center of Intelligent Mining Equipment, China University of Mining and Technology, No. 1 Daxue Road, \\ Xuzhou 221116, China \\ Correspondence should be addressed to Hongmei Wang; whm99@cumt.edu.cn
}

Received 23 December 2017; Accepted 25 March 2018; Published 23 April 2018

Academic Editor: Mucheol Kim

Copyright (C) 2018 Faguang Wang et al. This is an open access article distributed under the Creative Commons Attribution License, which permits unrestricted use, distribution, and reproduction in any medium, provided the original work is properly cited.

\begin{abstract}
A minimally invasive surgery robot is difficult to control when actuator saturation exists. In this paper, a Takagi-Sugeno fuzzy model-based controller is designed for a minimally invasive surgery robot with actuator saturation, which is difficult to control. The contractively invariant ellipsoid theorem is applied for the actuator saturation. The proposed scheme can be derived using the $\mathrm{H}$-infinity control theorem and parallel distributed compensation. The result is rebuilt in the form of linear matrix inequalities for easier calculation by computer. Meanwhile, the uniformly ultimately bounded stable and the prescribed $\mathrm{H}$-infinity control performance can be guaranteed. The proposed scheme is simulated in a Novint Falcon haptic device system.
\end{abstract}

\section{Introduction}

In minimally invasive robotic surgery, the work space is limited precisely. The mechanical structure and the electrical characteristics can be additional constraint boundaries for system inputs and outputs. Therefore, the controller of the surgery robot has a rigid input saturation requirement. However, if input saturation occurs, the output performance of the controlled object cannot satisfy the designed requirement, which can result in the decline of the closed-loop system response. The output overshoot cannot be suppressed well, even becoming unstable $[1,2]$. This situation is prohibited in a minimally invasive robotic surgery.

Many researchers investigated the input saturation problem and provided some solutions. Buckley [3] proposed an anti-reset windup method for the integral saturation problem. The error between the controller output and object input was used as a complementary feedback for the controlled system. Hanus et al. [4] proposed the condition technique, where the controller output continuously tracks for a new reference input and is located out of the saturation area to avoid the saturation case. The pole-placement method was applied to the antiwindup control, which assigns the poles of nonlinear system in the desired disk for stable analysis in [5]. Although the variable structure antiwindup controller showed a good performance in the integrator windup case [6], some preset parameters were needed that were difficult to determine because expert knowledge was required. Some antiwindup controllers were also based on observer [7], internal model control [8], saturation feedback control [9], and dynamic complement [10].

The T-S fuzzy theorem, which is an important part of the fuzzy control theorem, was proposed by Takagi and Sugeno in 1985 [11]. It is utilized in both system stable control and 
model identification $[11,12]$. Specifically, it has a good performance in nonlinear system control [12]. The T-S fuzzy model, which is the summation of the product of the T-S fuzzy local models and their corresponding membership functions, can approximate the nonlinear system under an arbitrary degree of accuracy [12]. The T-S fuzzy model is nonlinear, and its controller design is difficult. Consequently, a procedure called parallel distributed compensation (PDC) makes the T-S fuzzy controller design easier. PDC was proposed by Wang et al. in 1995 [13]. The fuzzy sets of the PDC controller are similar with the fuzzy model. Under each controller rule, a controller can be designed for the local T-S model. By summation of the product of these controllers and their corresponding membership functions, the total controller of a nonlinear system can be represented. System stability is usually considered by the Lyapunov function. Although PDC provides a design procedure for the T-S fuzzy controller, the calculation of controller gains for global stability is still difficult, particularly in the presence of many controller rules. A numerical optimization method called linear matrix inequalities (LMIs) solved this kind of problem. LMI was defined by Willems in 1971 [14]. Neestorv and Nemirovskii [15] proposed an interior point method, which can directly solve the LMI convex optimization problem. LMI was first applied to the T-S fuzzy system stabilization analysis in [16]. In the succeeding years, LMI became the focus of increasing number of researchers [17]. Except for a few special cases without analytical solution, LMIs are generally efficient [11]. Furthermore, the LMI toolbox software provides a direct shortcut to the computer solution of LMIs. Because PDC and LMIs provide a better T-S fuzzy system controller design, they are applied in the proposed controller design in this paper.

For the advantage of the T-S fuzzy theorem, it was widely used to deal with the actuator saturation problem. In [18], based on the T-S fuzzy model, a robust dissipative controller was designed for the multiple-input multipleoutput (MIMO) system with saturated time-delay input and parameter uncertainty. Their results show that the closedloop system can be stable, but the stabilization time cannot be guaranteed. For this problem, $[19,20]$ provided a finitetime control by optimal control and estimated the attraction domain. The combination of the T-S fuzzy model and optimal control show an animated controller design of a nonlinear system with actuator saturation [21]. For optimal control, $\mathrm{Hu}$ et al. [22] provided a useful control method for the saturation system with actuator saturation based on a contractively invariant ellipsoid. This study was continued in her work by BMIs [8]. Consequently, many researchers focused on actuator saturation problems, where the T-S fuzzy based controller design method was popular. The Lyapunov stability criterion-based PDC fuzzy controller was designed for the actuator saturation system [23, 24]. Many researchers contributed in completing this theorem $[25,26]$. In this paper, the result in $\mathrm{Hu}$ et al. [22] was adopted and rebuilt into a set of LMIs. Meanwhile, a predetermined $H_{\infty}$ norm was satisfied.

This paper is organized as follows. The basic T-S fuzzy theorem is presented in Section 2. The proposed solution for the actuator saturation problem is presented in Section
3. The simulation is presented in Section 4. The paper concludes in Section 5.

\section{General T-S Fuzzy Model and Control}

In this paper, the considered nonlinear system with disturbance $W(t)$ is

$$
\dot{X}(t)=f(X(t))+g(X(t)) U(t)+W(t),
$$

where $X(t) \in R^{n \times 1}, f(X(t)) \in R^{n \times 1}, g(X(t)) \in R^{n \times m}, U(t) \in$ $R^{m \times 1}$, and $\|W(t)\| \leq W_{\mathrm{b}}$ and $W_{\mathrm{b}}$ is the boundary of disturbance. In the T-S fuzzy model, the premise variables of the $\mathrm{T}$-S fuzzy rules must be measurable, and they can represent some properties of the nonlinear system. Therefore, a suitable selection of these variables is very important for the accuracy and reliability of the T-S fuzzy model. When the T-S fuzzy premise variables have been defined, a corresponding local model can be provided by these variable values. The $i$ th rule of the T-S fuzzy system for a nonlinear system is as follows:

The $i$ th rule: If $z_{1}(t)$ is $M_{i 1}, \ldots$, and $z_{p}(t)$ is $M_{i p}$, then

$$
\dot{X}(t)=A_{i} X(t)+B_{i} U(t)
$$

where $i=1,2, \ldots, L$ is the rule number, $\mathbf{A}_{i} \in R^{n \times n}$ and $\mathbf{B}_{i} \in$ $R^{n \times m}$ are the local model parameter matrices, $z_{1}, z_{2}, \ldots, z_{p}$ are the premise variables, and $M_{i 1}, \ldots, M_{i p}$ are the fuzzy sets. The nonlinear system can be approximated by the overall T-S fuzzy system

$$
\dot{X}(t)=\sum_{i=1}^{L} h_{i}(Z(t))\left\{A_{i} X(t)+B_{i} U(t)\right\}
$$

where $Z(t)=\left[z_{1}(t) z_{2}(t) \cdots z_{p}(t)\right]$ and $h_{i}(Z(t))=\mu_{i}(Z(t)) /$ $\sum_{i=1}^{L} \mu_{i}(Z(t)), \mu_{i}(Z(t))=\prod_{j=1}^{p} \theta_{i j}\left(z_{j}(t)\right)$ in which $\theta_{i j}(Z(t))$ is the grade of the membership of $z_{j}(t)$ in $M_{i j}$. Note that the membership function should satisfy the following equation:

$$
\begin{aligned}
h_{i}(Z(t)) & \geq 0, \\
\sum_{i=1}^{L} h_{i}(Z(t)) & =1 .
\end{aligned}
$$

Currently, the nonlinear system of (1) is changed to the T-S fuzzy model (3). Because the local T-S model of (2) is linear, its feedback controller can be designed easily as

$$
U_{j}=K_{j} X(t)
$$

where $K_{j} \in R^{m \times n}$ and $j=1,2, \ldots, L$. By PDC and similar fuzzy rules of (2), the overall T-S fuzzy controller is designed as

$$
U(t)=\sum_{j=1}^{L} h_{j}(Z(t)) K_{j} X(t) .
$$




\section{3. $H_{\mathbf{\infty}}$ Robust Controller Design for Input Saturation}

The traditional T-S fuzzy theorem cannot provide results in the control problem for a nonlinear system with input saturation. In this study, the proposed method can solve the input saturation problem. The controlled closed-loop system can be uniformly ultimately bounded (UUB) stable, and the prescribed $H$-infinity norm can be guaranteed. Furthermore, the result is shown in LMIs, which can be directly solved by programming.

Considering the saturation and uncertainty, the nonlinear system of (1) can be

$$
\dot{X}(t)=f(X(t))+g(X(t)) \cdot \operatorname{Sat}(U(t))+W(t)
$$

where

$$
\operatorname{Sat}(U(t))= \begin{cases}\operatorname{sgn}(U(t)) \cdot b, & \|U(t)\|>b, \\ U(t), & \|U(t)\| \leq b,\end{cases}
$$

$b>0$, is the boundary value of the saturation function, and the other symbols are similar with (1). Following (3), the T-S fuzzy model can be

$$
\dot{X}(t)=\sum_{i=1}^{L} h_{i}\left[A_{i} X(t)+B_{i} \operatorname{Sat}(U(t))\right]
$$

where

$$
U(t)=\sum_{j=1}^{L} h_{j}(Z(t)) U_{j}(t)
$$

Based on the work of $\mathrm{Hu}$ et al. [1], for the feedback controller, $U_{j}(t)=K_{j} X(t)$, if the ellipsoid $\varepsilon(P, 1)$ is contractively invariant, and matrixes $\mathbf{P}>0, \mathbf{K}_{i}$, and $\mathbf{H}(m \times n$ matrix $)$ make the initial value of $X_{0} \in \varepsilon(P, 1)$ and

$$
\varepsilon(P, 1) \subset \ell(H)
$$

where the polyhedron $\ell(H):=\left\{X(t) \in R^{n}:\left|H_{j} X(t)\right| \leq 1, j \in\right.$ $[1, m]\}$ and $H_{j}$ is the $j$ th row of $H$. The saturation feedback controller $\operatorname{Sat}(U(t))$ satisfies

$$
\begin{aligned}
\operatorname{Sat}\left(U_{j}(t)\right)= & \operatorname{Sat}\left(K_{j} X(t)\right) \in \mathrm{co} \\
& \cdot\left\{D_{r} K_{j} X(t)+D_{r}^{-} H_{j} X(t), r \in\left[1,2^{m}\right]\right\},
\end{aligned}
$$

where $D_{r}$ is an $m$-by- $m$ diagonal matrix (diagonal elements are 0 or 1$)$ and $D_{r}^{-}=I-D_{r}$ with $r \in\left[1,2^{m}\right]$.

If it is only applied to the T-S fuzzy local model, the global stability of the nonlinear system cannot be satisfied. For global stability, some changes are needed. By (7) and (9), the nonlinear system with disturbance is as follows:

$$
\begin{aligned}
\dot{X}(t)= & f(X(t))+g(X(t)) \operatorname{Sat}(U(t))+W(t) \\
= & \sum_{i=1}^{L} h_{i}(Z(t)) \cdot\left(A_{i} X(t)+B_{i} \operatorname{Sat}(U(t))\right)+f(X(t)) \\
& \quad-\sum_{i=1}^{L} h_{i}(Z(t)) A_{i} \cdot X(t) \\
& +\left(g(X(t))-\sum_{i=1}^{L} h_{i}(Z(t)) B_{i}\right) \operatorname{Sat}(U(t))+W(t) .
\end{aligned}
$$

Equation (12) can be guaranteed if each $D_{r}$ satisfies the following inequality:

$$
\begin{aligned}
& \dot{X} \leq \sum_{i=1}^{L} h_{i}(Z(t))\left(A_{i} X(t)+B_{i} \sum_{j=1}^{L} h_{j}(Z(t))\left(D_{r} K_{j} X(t)+D_{r}^{-} H_{j} X(t)\right)\right) \\
&+\left(f(X(t))-\sum_{i=1}^{L} h_{i}(Z(t)) A_{i} X(t)\right) \\
&+\left(g(X(t))-\sum_{i=1}^{L} h_{i}(Z(t)) B_{i}\right) \\
& \cdot\left(\sum_{j=1}^{L} h_{j}(Z(t))\left(D_{r} K_{j} X(t)+D_{r}^{-} H_{j} X(t)\right)\right)+W(t) \\
&=\sum_{i=1}^{L} \sum_{j=1}^{L} h_{i}(Z(t)) h_{j}(Z(t))\left(A_{i}+B_{i}\left(D_{r} K_{j}+D_{r}^{-} H_{j}\right)\right) X(t) \\
&+\Delta f+\Delta g+W(t),
\end{aligned}
$$

where

$$
\begin{gathered}
\Delta f=\left(f(X(t))-\sum_{i=1}^{L} h_{i}(Z(t)) A_{i} X(t)\right), \\
\Delta g=\sum_{i=1}^{L} h_{i}(Z(t)) \sum_{j=1}^{L} h_{j}(Z(t))\left(g(X(t))-B_{i}\right) \\
\cdot\left(D_{r} K_{j}+D_{r}^{-} H_{j}\right) X(t) .
\end{gathered}
$$

Some $\left\|\delta_{A i}\right\| \leq 1,\left\|\delta_{B i}\right\| \leq 1, A_{t}$, and $B_{t}$ can be found and satisfy the following inequality:

$$
\begin{aligned}
\|\Delta f\| & =\left\|\sum_{i=1}^{L} h_{i}(Z(t)) \Delta A_{i} X(t)\right\| \leq\left\|\sum_{i=1}^{L} h_{i}(Z(t)) \delta_{A i} A_{t} X(t)\right\| \\
& \leq\left\|A_{t} X(t)\right\| \\
\|\Delta g\| & \leq\left\|\sum_{j=1}^{L} h_{j}(Z(t)) B_{t}\left(D_{r} K_{j}+D_{r}^{-} H_{j}\right) X(t)\right\|
\end{aligned}
$$

where $\Delta A_{i}=\delta_{A i} A_{t}$ and $\Delta B_{i}=\delta_{B i} B_{t}$.

$H_{\infty}$ control performance is defined as

$$
\frac{\int_{0}^{t_{f}} X^{T}(t) Q X(t) d t}{\int_{0}^{t_{f}} W^{T}(t) W(t) d t}<\rho,
$$


where $t_{\mathrm{f}}$ is the terminal time, $\mathbf{Q}$ is a positive-definite matrix, and $\rho$ is the prescribed $H_{\infty}$ norm, which is greater than 0 and less than 1. If $\rho$ is minimized, the effect of $W(t)$ on $X(t)$ is minimized. Considering the initial condition, (17) can be changed as

$$
\int_{0}^{t_{f}} X^{T}(t) Q X(t) d t<X^{T}(0) P X(0)+\rho \int_{0}^{t_{f}} W^{T}(t) W(t) d t
$$

where $\mathbf{P}$ is some symmetric positive-definite weighting matrix. Set the Lyapunov function as

$$
V(t)=X^{T}(t) P X(t)
$$

where $\mathbf{P}>0$. For simplicity, $(t)$ is omitted in the following sections. Then, the following theorem can be obtained.

Theorem 1. Under condition (11), if controller (12) is applied to a nonlinear system (7) and there exists a positive-definite matrix $\mathbf{P}>0$, such that the following matrix inequalities

$$
\begin{aligned}
P A_{i} & +A_{i}^{T} P+P B_{i}\left(D_{r} K_{j}+D_{r}^{-} H_{j}\right)+\left(D_{r} K_{j}+D_{r}^{-} H_{j}\right)^{T} \\
& \cdot B_{i}{ }^{T} P+A_{t}{ }^{T} A_{t}+\left(D_{r} K_{j}+D_{r}^{-} H_{j}\right)^{T} B_{t}{ }^{T} B_{t}\left(D_{r} K_{j}+D_{r}^{-} H_{j}\right) \\
& +2 P P+\rho^{-2} P P+Q_{i}<0
\end{aligned}
$$

are satisfied for each $i, j$, and $r$, then the closed-loop system is $U U B$, and the $H_{\infty}$ control performance (18) is guaranteed as prescribed $\rho^{2}$.

Proof 1. Using (14), the derivative of $V(t)$ is

$$
\begin{aligned}
& \dot{V}(t)= X^{T} P \dot{X}+\dot{X}^{T} P X=X^{T} P\left\{\sum_{I=1}^{l} h_{i}\left[A_{i} X+B_{i} \operatorname{Sat}(U)+W\right]\right\} \\
&+\left\{\sum_{i=1}^{l} h_{i}\left[A_{i} X+B_{i} \operatorname{Sat}(U)+W\right]\right\}^{T} P X \leq X^{T} P \\
& \cdot\left\{\sum_{i=1}^{L} \sum_{j=1}^{L} h_{i} h_{j}\left(A_{i}+B_{i}\left(D_{r} K_{j}+D_{r}^{-} H_{j}\right)\right) X+\Delta f+\Delta g+W\right\} \\
&+\left\{\sum_{i=1}^{L} \sum_{j=1}^{L} h_{i} h_{j}\left(A_{i}+B_{i}\left(D_{r} K_{j}+D_{r}^{-} H_{j}\right)\right) X+\Delta f+\Delta g+W\right\}^{T} \\
& \cdot P X \leq \sum_{i=1}^{L} \sum_{j=1}^{L} h_{i} h_{j}\left\{X ^ { T } \left(P\left(A_{i}+B_{i}\left(D_{r} K_{j}+D_{r}^{-} H_{j}\right)\right)\right.\right. \\
&\left.+\left(A_{i}+B_{i}\left(D_{r} K_{j}+D_{r}^{-} H_{j}\right)\right)^{T} P\right) X \\
&+(\Delta f)^{T}(\Delta f)+X^{T} P P X+(\Delta g)^{T}(\Delta g) \\
&\left.+X^{T} P P X\right\}+X^{T} P W+W^{T} P X .
\end{aligned}
$$

$$
\begin{aligned}
& \dot{V} \leq \sum_{i=1}^{L} \sum_{j=1}^{L} h_{i} h_{j}\left\{X ^ { T } \left(P\left(A_{i}+B_{i}\left(D_{r} K_{j}+D_{r}^{-} H_{j}\right)\right)\right.\right. \\
&\left.+\left(A_{i}+B_{i}\left(D_{r} K_{j}+D_{r}^{-} H_{j}\right)\right)^{T} P\right) X+\left(A_{t} X\right)^{T}\left(A_{t} X\right) \\
&+X^{T} P P X+\left(B_{t}\left(D_{r} K_{j}+D_{r}^{-} H_{j}\right) X\right)^{T} \\
&\left.+\left(B_{t}\left(D_{r} K_{j}+D_{r}^{-} H_{j}\right) X\right)+X^{T} P P X\right\}+X^{T} P W \\
&+W^{T} P X \\
&=\sum_{i=1}^{L} \sum_{j=1}^{L} h_{i} h_{j} X^{T}\left\{\left(P A_{i}+A_{i}^{T} P+P B_{i}\left(D_{r} K_{j}+D_{r}^{-} H_{j}\right)\right.\right. \\
&+\left(D_{r} K_{j}+D_{r}^{-} H_{j}\right)^{T} B_{i}{ }^{T} P+A_{t}{ }^{T} A_{t}+\left(D_{r} K_{j}+D_{r}^{-} H_{j}\right)^{T} \\
&\left.\left.\cdot B_{t}{ }^{T} B_{t}\left(D_{r} K_{j}+D_{r}^{-} H_{j}\right)+2 P P\right)\right\} X-\left(\rho^{-1} P X-\rho W\right)^{T} \\
& \cdot\left(\rho^{-1} P X-\rho W\right)+\rho^{2} W^{T} W+\rho^{-2} X^{T} P P X \\
& \leq \sum_{i=1}^{L} \sum_{j=1}^{L} h_{i} h_{j} X^{T}\left\{\left(P A_{i}+A_{i}^{T} P+P B_{i}\left(D_{r} K_{j}+D_{r}^{-} H_{j}\right)\right.\right. \\
&+\left(D_{r} K_{j}+D_{r}^{-} H_{j}\right)^{T} B_{i}{ }^{T} P+A_{t}{ }^{T} A_{t} \\
&+\left(D_{r} K_{j}+D_{r}^{-} H_{j}\right)^{T} B_{t}{ }^{T} B_{t} \cdot\left(D_{r} K_{j}+D_{r}^{-} H_{j}\right) \\
&++2 P P)\} X+\rho^{2} W^{T} W+\rho^{-2} X^{T} P P X .
\end{aligned}
$$

Under the $i$ th rule, if there is

$$
\frac{\int_{0}^{t_{\mathrm{f}}} X^{T}(t)\left(h_{i}^{2} Q_{i}\right) X(t) d t}{\int_{0}^{t_{\mathrm{f}}} W^{T}(t) W(t) d t} \leq \frac{\int_{0}^{t_{\mathrm{f}}} X^{T}(t) Q_{i} X(t) d t}{\int_{0}^{t_{\mathrm{f}}} W^{T}(t) W(t) d t}<\rho,
$$

where $Q_{i}>0$ and $\sum_{i=1}^{L}\left(h_{i} Q_{i}\right)>0$, there is

$$
\frac{\int_{0}^{t_{f}} X^{T}(t)\left(\sum_{i=1}^{L} h_{i} Q_{i}\right) X(t) d t}{\int_{0}^{t_{f}} W^{T}(t) W(t) d t}<\rho
$$

for the nonlinear system. If $\sum_{i=1}^{L}\left(h_{i} Q_{i}\right)=Q$, (24) can be the $H_{\infty}$ control performance defined in (17). Therefore, for each simulation time, the $H_{\infty}$ control performance defined as (17) can be guaranteed. Then, in the total simulation, a prescribed $H_{\infty}$ norm can be guaranteed. Meanwhile, (18) can be as follows:

$$
\begin{aligned}
& \int_{t=0}^{t=t_{\mathrm{f}}}\left(X^{T}\left(\sum_{i=1}^{L} h_{i} Q_{i}\right) X\right) d t<X^{T}(0) P X(0)+\rho \int_{t=0}^{t=t_{\mathrm{f}}}\left(W^{T} W\right) d t . \\
& \text { If } \\
& \begin{aligned}
P A_{i} & +A_{i}^{T} P+P B_{i}\left(D_{r} K_{j}+D_{r}^{-} H\right)+\left(D_{r} K_{j}+D_{r}^{-} H\right)^{T} B_{i}{ }^{T} P \\
& +A_{t}{ }^{T} A_{t}+\left(D_{r} K_{j}+D_{r}^{-} H\right)^{T} B_{t}{ }^{T} B_{t}\left(D_{r} K_{j}+D_{r}^{-} H\right) \\
& +2 P P<-\rho^{-2} P P-Q_{i},
\end{aligned}
\end{aligned}
$$

Using (16), (21) can be

there is 


$$
\begin{gathered}
\dot{V}<\sum_{i=1}^{L} \sum_{j=1}^{L} h_{i} h_{j} X^{T}\left\{-\rho^{-2} P P-Q_{i}\right\} X+\rho^{2} W^{T} W+\rho^{-2} X^{T} P P X \\
=X^{T}\left\{-\rho^{-2} P P-\left(\sum_{i=1}^{L} h_{i} Q_{i}\right)\right\} X+\rho^{2} W^{T} W \\
+\rho^{-2} X^{T} P P X=-X^{T}\left(\sum_{i=1}^{L} h_{i} Q_{i}\right) X+\rho^{2} W^{T} W
\end{gathered}
$$

By integrating (27), the following result can be obtained:

$$
V\left(t_{\mathrm{f}}\right)-V(0)<\int_{t=0}^{t=t_{\mathrm{f}}}\left(-X^{T}\left(\sum_{i=1}^{L} h_{i} Q_{i}\right) X+\rho^{2} W^{T} W\right) d t .
$$

The Lyapunov function (19) can be 0 when the system is stable. Therefore,

$$
\int_{t=0}^{t=t_{\mathrm{f}}}\left(X^{T}\left(\sum_{i=1}^{L} h_{i} Q_{i}\right) X\right) d t<X^{T}(0) P X(0)+\rho^{2} \int_{t=0}^{t=t_{\mathrm{f}}}\left(W^{T} W\right) d t .
$$

The above equation is the $H_{\infty}$ performance defined in (25), which satisfies (18). Note that the $H_{\infty}$ norm is changed to $\rho^{2}$.

End of proof.

In Theorem 1, it is difficult to find the solution for (20). Therefore, the transformation of inequality (20) into LMIs is necessary. By setting $R=P^{-1}$ and left and right multiplication of $R,(20)$ can be

$$
\begin{aligned}
A_{i} R & +R A_{i}^{T}+B_{i}\left(D_{r} K_{j}+D_{r}^{-} H_{j}\right) R+R\left(D_{r} K_{j}+D_{r}^{-} H_{j}\right)^{T} B_{i}{ }^{T} \\
& +R A_{t}{ }^{T} A_{t} R+R\left(D_{r} K_{j}+D_{r}^{-} H_{j}\right)^{T} B_{t}{ }^{T} B_{t}\left(D_{r} K_{j}+D_{r}^{-} H_{j}\right) R \\
& +2+\rho^{-2}+R Q_{i} R<0 .
\end{aligned}
$$

Let $\widehat{K}_{i}=K_{i} \cdot R, \widehat{H}_{i}=H_{i} \cdot R$, and $\widehat{Q}_{i}=\left(A_{t}{ }^{T} A_{t}+Q_{i}\right)^{-1}$. By Schur complements, (30) can be changed as

$$
\left[\begin{array}{ccc}
A^{*} & \left(B_{t}\left(D_{r} \widehat{K}_{j}+D_{r}^{-} \widehat{H}_{j}\right)\right)^{T} & R \\
B_{t}\left(D_{r} \widehat{K}_{j}+D_{r}^{-} \widehat{H}_{j}\right) & -I & 0 \\
R & 0 & -\widehat{Q}_{i}
\end{array}\right]<0,
$$

where $A^{*}=A_{i} R+R A_{i}^{T}+B_{i}\left(D_{r} \widehat{K}_{j}+D_{r}^{-} \widehat{H}_{j}\right)+\left(D_{r} \widehat{K}_{j}+D_{r}^{-}\right.$. $\left.\widehat{H}_{j}\right)^{T} B_{i}{ }^{T}+\left(2+\rho^{-2}\right) I, R, \widehat{K}_{j}$, and $\widehat{H}_{j}$ are unknown. Condition (11) is equal to

$$
\left[\begin{array}{cc}
1 & \widehat{H}_{i k} \\
\widehat{H}_{i k}{ }^{T} & R
\end{array}\right] \geq 0
$$

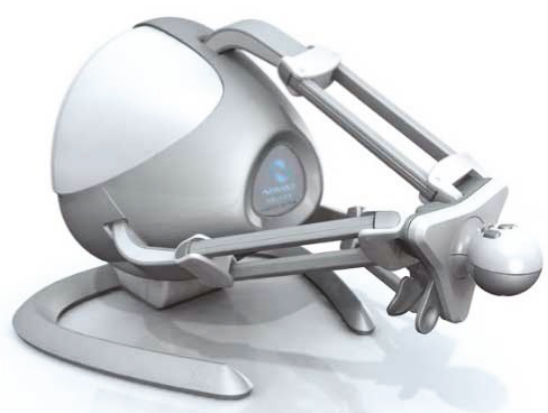

Figure 1: Novint Falcon.

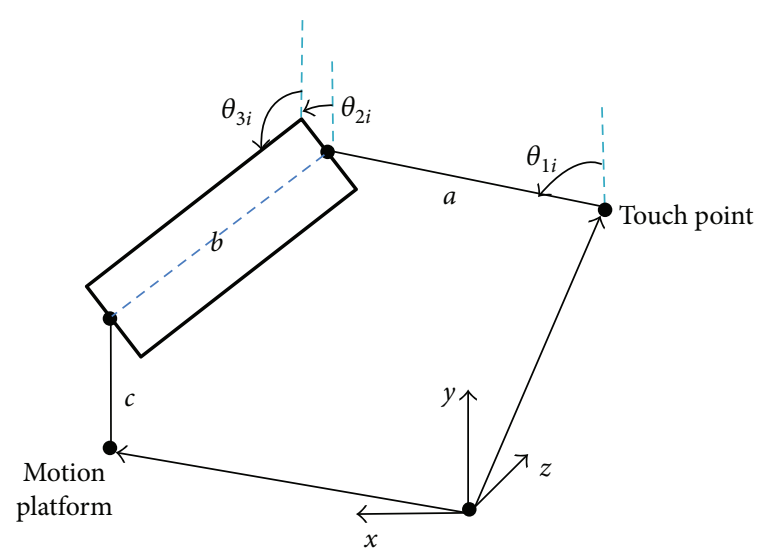

Figure 2: Mathematical model of the single chain.

where $\widehat{H}_{i k}$ is the $k$ th row of $\widehat{H}_{i}$. Therefore, the following theorem is given.

Theorem 2. For nonlinear system (7) with controller (12), if there exists

$\min \rho^{2}$,

subject to $\left[\begin{array}{ccc}A^{*} & \left(B_{t}\left(D_{r} \widehat{K}_{j}+D_{r}^{-} \widehat{H}_{j}\right)\right)^{T} & R \\ B_{t}\left(D_{r} \widehat{K}_{j}+D_{r}^{-} \widehat{H}_{j}\right) & -I & 0 \\ R & 0 & -\widehat{Q}_{i}\end{array}\right]<0$,

$\left[\begin{array}{cc}1 & \widehat{H}_{i k} \\ \hat{H}_{i k}{ }^{T} & R\end{array}\right] \geq 0$

$R>0$,

then the closed-loop system is UUB and the $H_{\infty}$ control performance (18) is guaranteed as prescribed $\rho^{2}$.

By this theorem, the $H_{\infty}$ optimization problem can be changed to a constrained optimization problem by LMIs. 

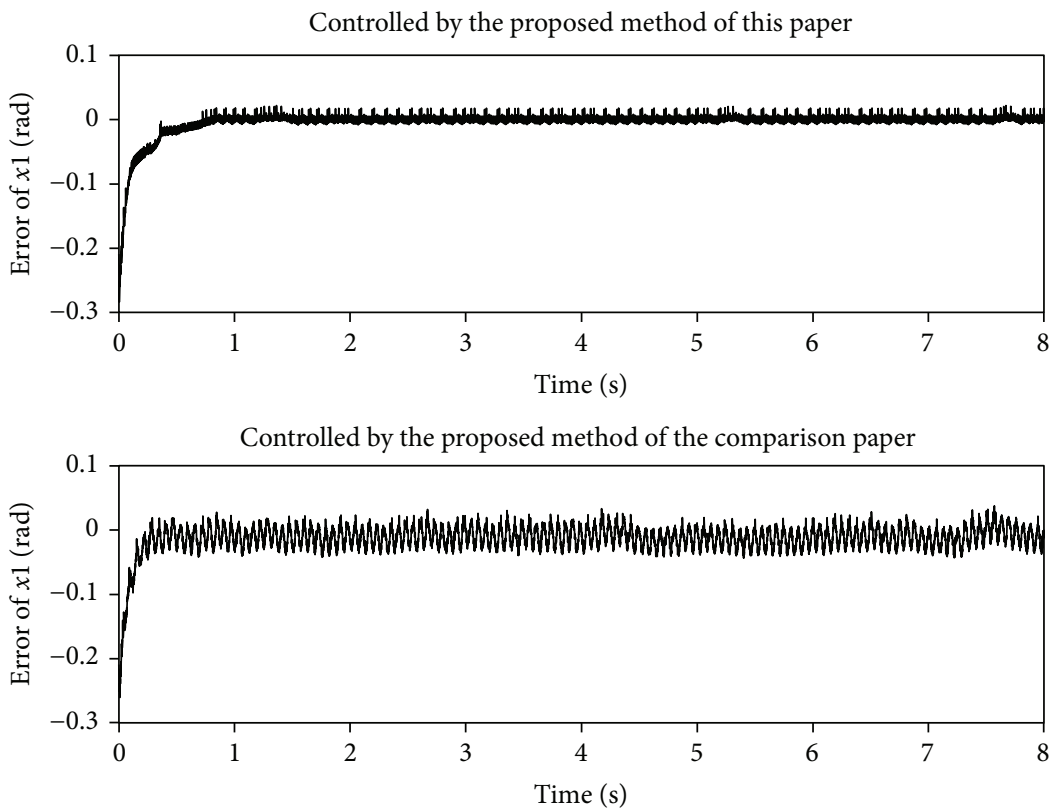

Figure 3: The error of $x_{1}$.

\section{Numerical Example}

In minimally invasive surgery, haptic tracking is very important. Novint Falcon is a simple arm robot with force feedback ability, which benefited from parallel architecture, and can be applied in minimally invasive surgery. The Novint Falcon structure is shown in Figure 1.

The Novint Falcon consists of the base, motion platform, and 3 chains. The single chain can be abstract presented in Figure 2.

Based on [27] and ignoring some nonessentials, the $i$ th chain dynamic equation is

$$
\tau=D(\theta) \ddot{\theta}+H(\theta, \dot{\theta}) \dot{\theta}+G(\theta)
$$

where $\tau=\left[\tau_{1}, \tau_{2}, \tau_{3}\right]^{T}$ is the moment of driving force for each link; $\theta=\left[\theta_{1 i}, \theta_{2 i}, \theta_{3 i}\right]^{T}$ is shown in Figure $2 ; \mathbf{D}(\theta)=I_{T}+$ $\left(J^{T}\right)^{-1}\left(3 m_{b}+m_{c}\right) J^{-1}$, where $I_{T}$ is the moment of inertia and $m_{b}$ and $m_{c}$ are masses of links $b$ and $c ; H(\theta, \dot{\theta})=D_{\mathrm{f}}+$ $\left(J^{T}\right)^{-1}\left(3 m_{b}+m_{c}\right)\left(\left(J^{T}\right)^{-1}\right)^{\prime}$, where $D_{\mathrm{f}}$ denotes the damped coefficient; $G(\theta)=-M_{\mathrm{g}}$ is the gravity moment;

$$
\mathbf{J}=\left[\begin{array}{lll}
J_{11} & J_{12} & J_{13} \\
J_{21} & J_{22} & J_{23} \\
J_{31} & J_{32} & J_{33}
\end{array}\right]^{-1}\left[\begin{array}{ccc}
\alpha_{1} & 0 & 0 \\
0 & \alpha_{2} & 0 \\
0 & 0 & \alpha_{3}
\end{array}\right]
$$

is the Jacobian matrix; $J_{i 1}=\cos \left(\theta_{2 i}\right) \sin \left(\theta_{3 i}\right) \cos \left(\phi_{i}\right)-\cos \left(\theta_{3 i}\right)$ $\sin \left(\phi_{i}\right), \quad J_{i 2}=\cos \left(\theta_{3 i}\right) \cos \left(\phi_{i}\right)+\cos \left(\theta_{2 i}\right) \sin \left(\theta_{3 i}\right) \sin \left(\phi_{i}\right), \quad J_{i 3}=$ $\sin \left(\theta_{2 i}\right) \sin \left(\theta_{3 i}\right), \alpha_{i}=\sin \left(\theta_{2 i}-\theta_{1 i}\right) \sin \left(\theta_{3 i}\right)$, and $\phi$ are the angle between the chain and the coordinate frame $(x, y, z)$. Because the exact parameters are difficult to determine, set $\mathbf{I}_{T}$ and $\mathbf{M}_{\mathrm{g}}$ as identity matrices and $3 m_{b}+m_{c}=1$ in this example. This is reasonable in this paper because the error between the actual value and the required value can be distributed in the disturbance or parameter uncertainty for this system. Therefore, the matrix $\mathbf{D}(\theta)$ is invertible. Set $\theta_{1 i}=x_{1}(t), \theta_{2 i}=x_{2}(t)$, $\theta_{3 i}=x_{3}(t), \tau=u(t)$, and

$$
\begin{aligned}
& \dot{x}_{1}(t)=x_{4}(t), \\
& \dot{x}_{2}(t)=x_{5}(t), \\
& \dot{x}_{3}(t)=x_{6}(t) .
\end{aligned}
$$

The $\theta$ of the other chains are set to be closed to require values, and the errors are distributed in the disturbance. Therefore, the system (34) can be as follows:

$$
\left[\begin{array}{l}
\dot{x}_{4}(t) \\
\dot{x}_{5}(t) \\
\dot{x}_{6}(t)
\end{array}\right]=A\left[\begin{array}{l}
x_{4}(t) \\
x_{5}(t) \\
x_{6}(t)
\end{array}\right]+B u(t)+E
$$

where $A=-D(\theta)^{-1} H(\theta, \dot{\theta}), \quad B=D(\theta)^{-1}$, and $E=-D(\theta)^{-1}$ $G(\theta)$. Considering actuator saturation, the corresponding $i$ th fuzzy rule is as follows:

Rule $i$. If $A$ is $A_{i}$ and $B$ is $B_{i}$, then (37) is

$$
\left[\begin{array}{l}
\dot{x}_{4}(t) \\
\dot{x}_{5}(t) \\
\dot{x}_{6}(t)
\end{array}\right]=A_{i}\left[\begin{array}{c}
x_{4}(t) \\
x_{5}(t) \\
x_{6}(t)
\end{array}\right]+B_{i} \operatorname{Sat}(u(t)),
$$

where $A_{1}=A\left(\max \left(\theta_{1 i}\right), \max \left(\theta_{2 i}\right), \max \left(\theta_{3 i}\right)\right), A_{2}=A\left(\min \left(\theta_{1 i}\right)\right.$, $\left.\max \left(\theta_{2 i}\right), \max \left(\theta_{3 i}\right)\right), \quad A_{3}=A\left(\max \left(\theta_{1 i}\right), \min \left(\theta_{2 i}\right), \max \left(\theta_{3 i}\right)\right)$, $A_{4}=A\left(\max \left(\theta_{1 i}\right), \max \left(\theta_{2 i}\right), \min \left(\theta_{3 i}\right)\right), A_{5}=A\left(\min \left(\theta_{1 i}\right), \min \right.$ $\left.\left(\theta_{2 i}\right), \max \left(\theta_{3 i}\right)\right), A_{6}=A\left(\min \left(\theta_{1 i}\right), \max \left(\theta_{2 i}\right), \min \left(\theta_{3 i}\right)\right), A_{7}=$ 

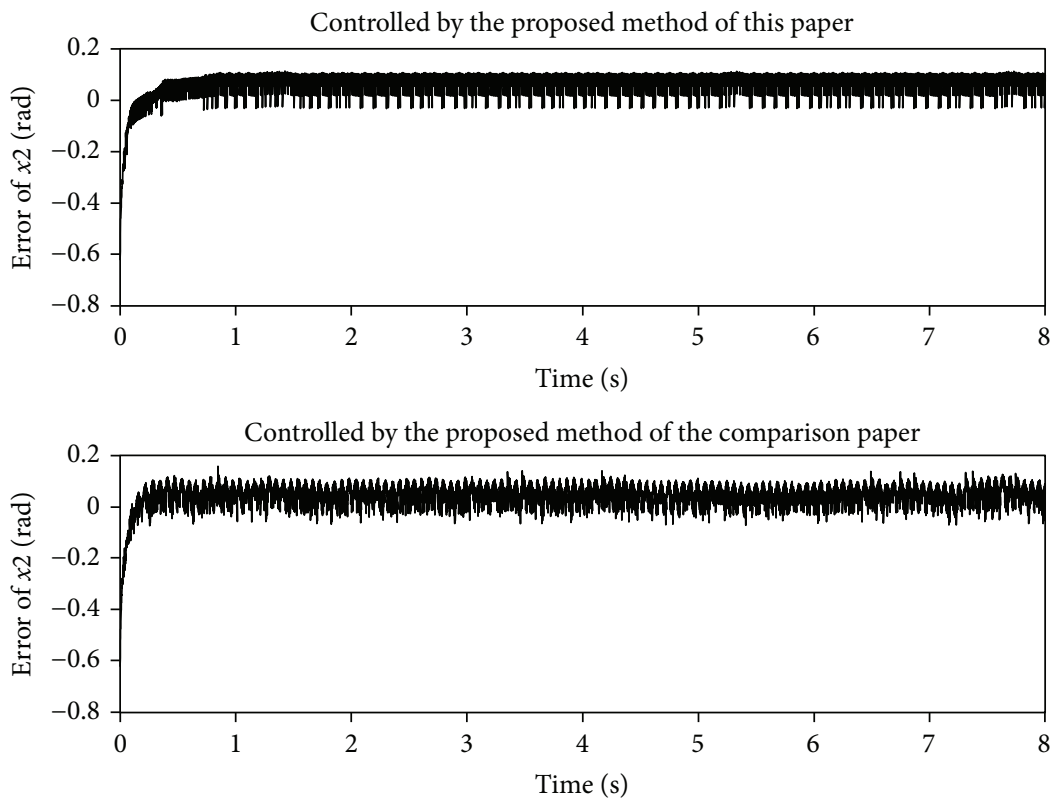

FIgURE 4: The error of $x_{2}$.

$A\left(\max \left(\theta_{1 i}\right), \min \left(\theta_{2 i}\right), \min \left(\theta_{3 i}\right)\right)$, and $A_{8}=A\left(\min \left(\theta_{1 i}\right), \min \right.$ $\left.\left(\theta_{2 i}\right), \min \left(\theta_{3 i}\right)\right) ; B_{i}$ is calculated similarly, and $E$ is treated as a disturbance. Although $A_{i}$ depends on $\theta$, the values of $\max (\theta)$ and $\min (\theta)$ do not depend on the system input. Therefore, they can be used for the fuzzy rule definition. Set $\left.\theta_{1 i} \in[0,0.7854], \theta_{2 i} \in[0,0.7854]\right]$, and $\theta_{3 i} \in[1.9199,2.2689]$.
$\operatorname{Sat}(u(t))=\operatorname{sgn}(u(t)) \cdot \min \{|u(t)|, 10\}$. The $H_{\infty}$ norm is 0.3 . The reference value is

$$
\left[\begin{array}{llllll}
x_{1 r} & x_{2 r} & x_{3 r} & \mathrm{x}_{4 r} & x_{5 r} & x_{6 r}
\end{array}\right]=\left[\begin{array}{llllll}
0.7 & 0.7 & 2.2 & 0 & 0 & 0
\end{array}\right] \text {. }
$$

Using Theorem 2, the error system feedback controller gains can be calculated as follows:

$$
\begin{aligned}
& \mathrm{K} 1=\left[-2.9 \times 10^{4},-1.8 \times 10^{5}, 2.6 \times 10^{4} ; 3.0 \times 10^{4}, 6.5 \times 10^{4},-3.4 \times 10^{4} ;-7.9 \times 10^{2}, 1.2 \times 10^{5}, 7.7 \times 10^{3}\right], \\
& \mathrm{K} 2=\left[1.8 \times 10^{4}, 1.6 \times 10^{5},-1.4 \times 10^{4} ;-1.8 \times 10^{4},-3.2 \times 10^{4}, 2.1 \times 10^{4} ;-1.2 \times 10^{2},-1.3 \times 10^{5},-6.9 \times 10^{3}\right], \\
& \mathrm{K} 3=\left[-3.8 \times 10^{4},-4.7 \times 10^{5}, 2.2 \times 10^{4} ; 1.4 \times 10^{4}, 1.1 \times 10^{5},-1.2 \times 10^{4} ; 2.4 \times 10^{4}, 3.6 \times 10^{5},-1.1 \times 10^{4}\right], \\
& \mathrm{K} 4=\left[1.9 \times 10^{4}, 2.9 \times 10^{5},-7.6 \times 10^{3} ;-1.7 \times 10^{4}, 2.2 \times 10^{3}, 2.2 \times 10^{4} ;-1.4 \times 10^{3},-2.9 \times 10^{5},-1.4 \times 10^{4}\right], \\
& \mathrm{K} 5=\left[-2.0 \times 10^{4},-2.4 \times 10^{5}, 1.2 \times 10^{4} ; 9.7 \times 10^{3}, 5.1 \times 10^{4},-9.5 \times 10^{3} ; 9.8 \times 10^{3}, 1.8 \times 10^{5},-2.1 \times 10^{3}\right], \\
& \mathrm{K} 6=\left[8.4 \times 10^{3}, 1.3 \times 10^{5},-3.6 \times 10^{3} ;-7.5 \times 10^{3},-3.2 \times 10^{3}, 9.3 \times 10^{3} ;-9.1 \times 10^{2},-1.2 \times 10^{5},-5.7 \times 10^{3}\right], \\
& \mathrm{K} 7=\left[-1.9 \times 10^{3},-3.0 \times 10^{5},-1.4 \times 10^{4} ; 4.4 \times 10^{3},-1.1 \times 10^{5},-1.1 \times 10^{4} ;-2.4 \times 10^{3}, 4.0 \times 10^{5}, 2.5 \times 10^{4}\right], \\
& \mathrm{K} 8=\left[9.2 \times 10^{2}, 6.5 \times 10^{4}, 2.4 \times 10^{3} ;-2.1 \times 10^{3}, 1.9 \times 10^{4}, 3.6 \times 10^{3} ; 1.1 \times 10^{3},-8.4 \times 10^{4},-6.0 \times 10^{3}\right] .
\end{aligned}
$$

To test the robustness of the controller, a disturbance

$$
\left.W(t)=\left[\sin \frac{(12 t)}{3} \quad 0 \quad 0\right]\right]^{T}
$$

is added in (38). For comparison, a controller designed by Theorem 2 of [18] is applied. In [18], the controller was designed for the system with input saturation and disturbance. If the time delay is ignored in [18], its results can be applied in this example. The simulation results are shown in the following figures.
From Figures 3-5, less overshooting can be observed compared with the reference paper. The proposed controller performed well with input saturation, and the $H_{\infty}$ performance can be achieved.

\section{Conclusion}

In this paper, the T-S fuzzy control theorem and the ability of contractively invariant ellipsoid were applied for nonlinear systems with input saturation. The proposed method was 

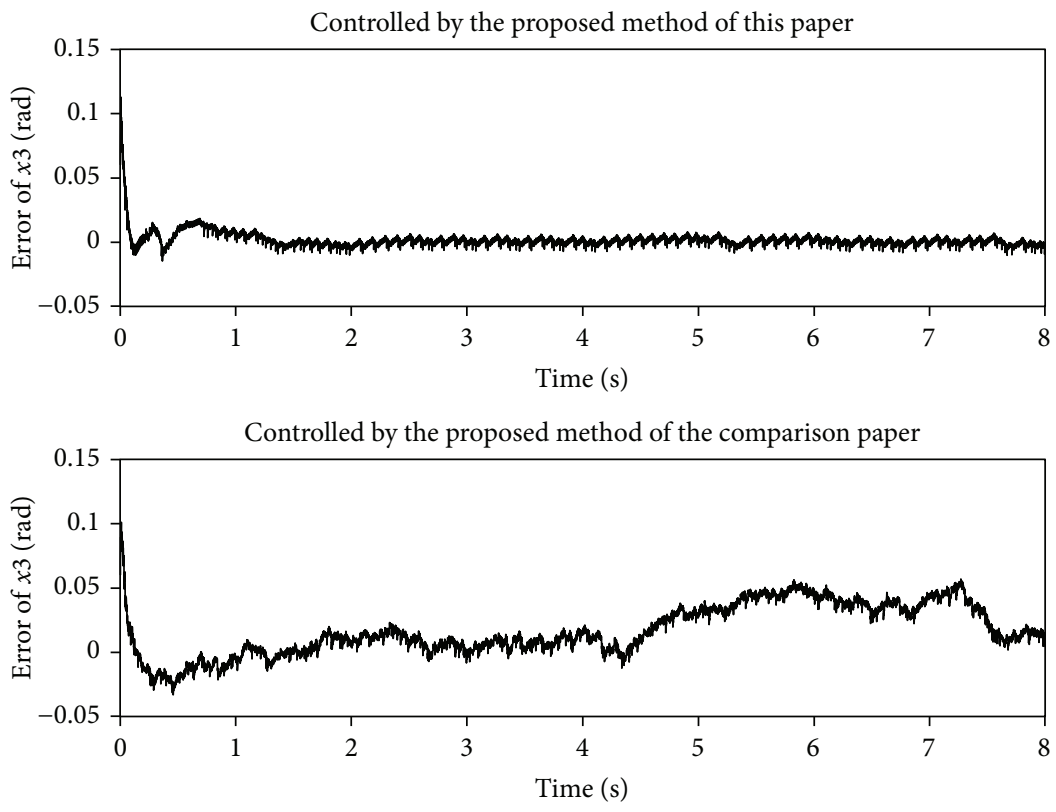

Figure 5: The error of $x_{3}$.

described by LMIs, which can be calculated by a computer. The proposed theorem was tested using the Novint Falcon, and a good result was achieved. Comparing the results, the proposed method of this paper has given a better result. Meanwhile, the prescribed $H$-infinity norm was satisfied. In the example, only eight rules of the T-S fuzzy system were applied; there were still more than one hundred LMIs that need to be calculated. Although a feasible solution of the LMIs can be guaranteed using the proposed method, a method with less LMIs is still necessary for further study.

\section{Conflicts of Interest}

The authors declare that they have no conflicts of interest.

\section{Acknowledgments}

This work was supported in part by the National Natural Science Foundation of China (61601464) and the Fundamental Research Funds for the Central Universities (2013QNB26).

\section{References}

[1] T. Hu, A. R. Teel, and L. Zaccarian, "Stability and performance for saturated systems via quadratic and nonquadratic Lyapunov functions," IEEE Transactions on Automatic Control, vol. 51, no. 11, pp. 1770-1786, 2006.

[2] S. Sajjadi-Kia and F. Jabbari, "Controllers for linear systems with bounded actuators: slab scheduling and anti-windup," Automatica, vol. 49, no. 3, pp. 762-769, 2013.

[3] P. Buckley, "Designing override and feedforward controls," Control Engineering, vol. 18, no. 8, pp. 48-51, 1971.

[4] R. Hanus, M. Kinnaert, and J.-L. Henrotte, "Conditioning technique, a general anti-windup and bumpless transfer method," Automatica, vol. 23, no. 6, pp. 729-739, 1987.
[5] F.-G. Wang, H.-M. Wang, S.-K. Park, and X.-S. Wang, "Linear pole-placement anti-windup control for input saturation nonlinear system based on Takagi Sugeno fuzzy model," International Journal of Control, Automation and Systems, vol. 14, no. 6, pp. 1599-1606, 2016.

[6] V. D. Hajare, A. A. Khandekar, and B. M. Patre, "Discrete sliding mode controller with reaching phase elimination for TITO systems," ISA Transactions, vol. 66, pp. 32-45, 2017.

[7] W. Liu, J. Lu, Z. Zhang, and S. Xu, "Observer-based neural control for MIMO pure-feedback non-linear systems with input saturation and disturbances," IET Control Theory \& Applications, vol. 10, no. 17, pp. 2314-2324, 2016.

[8] A. Lekka, M. C. Turner, and P. P. Menon, "Anti-windup for a class of partially linearisable non-linear systems with application to wave energy converter control," IET Control Theory \& Applications, vol. 10, no. 18, pp. 2403-2414, 2016.

[9] C. Hua, Y. Yang, and P. X. Liu, "Output-feedback adaptive control of networked teleoperation system with time-varying delay and bounded inputs," IEEE/ASME Transactions on Mechatronics, vol. 20, no. 5, pp. 2009-2020, 2015.

[10] L. Zaccarian, Y. Li, E. Weyer, M. Cantoni, and A. R. Teel, "Anti-windup for marginally stable plants and its application to open water channel control systems," Control Engineering Practice, vol. 15, no. 2, pp. 261-272, 2007.

[11] T. Takagi and M. Sugeno, "Fuzzy identification of systems and its applications to modeling and control," IEEE Transactions on Systems, Man, and Cybernetics, vol. SMC-15, no. 1, pp. 116-132, 1985.

[12] K. Tanaka and H. O. Wang, Fuzzy Control Systems Design and Analysis, John Wiley \& Sons Inc., New York, NY, USA, 2001.

[13] H. O. Wang, K. Tanaka, and M. F. Griffin, "Parallel distributed compensation of nonlinear systems by Takagi-Sugeno fuzzy model," in Proceedings of 1995 IEEE International Conference on Fuzzy Systems, pp. 531-538, Yokohama, Japan, 1995. 
[14] J. Willems, "Least squares stationary optimal control and the algebraic Riccati equation," IEEE Transactions on Automatic Control, vol. 16, no. 6, pp. 621-634, 1971.

[15] Y. Neestorv and A. Nemirovskii, "A general approach to polynomial-time algorithms design for convex programming," Technical Report, Central Economical and Mathematical Institute, USSR, Moscow, Russia, 1998.

[16] K. Tanaka, T. Ikeda, and H. O. Wang, "Robust stabilization of a class of uncertain nonlinear systems via fuzzy control: quadratic stabilizability, $\mathrm{H}_{\infty}$ control theory, and linear matrix inequalities," IEEE Transactions on Fuzzy Systems, vol. 4, no. 1, pp. 1-13, 1996.

[17] S. Xing, Q. Zhang, and B. Zhu, "Mean-square admissibility for stochastic T-S fuzzy singular systems based on extended quadratic Lyapunov function approach," Fuzzy Sets and Systems, vol. 307, pp. 99-114, 2017.

[18] S. Xu, G. Sun, and W. Sun, "Takagi-Sugeno fuzzy model based robust dissipative control for uncertain flexible spacecraft with saturated time-delay input," ISA Transactions, vol. 66, pp. 105-121, 2017.

[19] W. Guan and F. Liu, "Finite-time dissipative control for singular T-S fuzzy Markovian jump systems under actuator saturation with partly unknown transition rates," Neurocomputing, vol. 207, pp. 60-70, 2016.

[20] W. Guan and F. Liu, "Finite-time $H_{\infty}$ memory state feedback control for uncertain singular TS fuzzy time-delay system under actuator saturation," Advances in Difference Equations, vol. 2016, no. 1, 19 pages, 2016.

[21] Y. Ma, P. Yang, and Q. Zhang, "Robust $\mathrm{H}_{\infty}$ control for uncertain singular discrete T-S fuzzy time-delay systems with actuator saturation," Journal of the Franklin Institute, vol. 353, no. 13, pp. 3290-3311, 2016.

[22] T. Hu, Z. Lin, and B. M. Chen, "Analysis and design for discrete-time linear systems subject to actuator saturation," Systems \& Control Letters, vol. 45, no. 2, pp. 97-112, 2002.

[23] W.-J. Chang and Y.-J. Shih, "Fuzzy control of multiplicative noised nonlinear systems subject to actuator saturation and $H_{\infty}$ performance constraints," Neurocomputing, vol. 148, pp. 512-520, 2015.

[24] S. Wen and Y. Yan, "Robust adaptive fuzzy control for a class of uncertain MIMO nonlinear systems with input saturation," Mathematical Problems in Engineering, vol. 2015, Article ID 561397, 14 pages, 2015.

[25] A. Nguyen, T. Laurain, R. Palhares, J. Lauber, C. Sentouh, and J. Popieul, "LMI-based control synthesis of constrained Takagi-Sugeno fuzzy systems subject to $\mathscr{L}_{2}$ or $\mathscr{L}_{\infty}$ disturbances," Neurocomputing, vol. 207, pp. 793-804, 2016.

[26] S. Gao, H. Dong, B. Ning, and X. Yao, "Single-parameterlearning-based fuzzy fault-tolerant output feedback dynamic surface control of constrained-input nonlinear systems," Information Sciences, vol. 385-386, pp. 378-394, 2017.

[27] N. Karbasizadeh, A. Aflakiyan, M. Zarei, M. T. Masoulehy, and A. Kalhor, "Dynamic identification of the Novint Falcon haptic device," in Proceedings of 4-th International Conference on Robotics and Mechatronics, Tehran, Iran, 2016. 


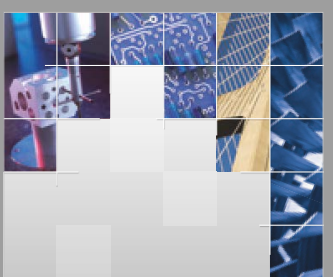

\section{Enfincering}
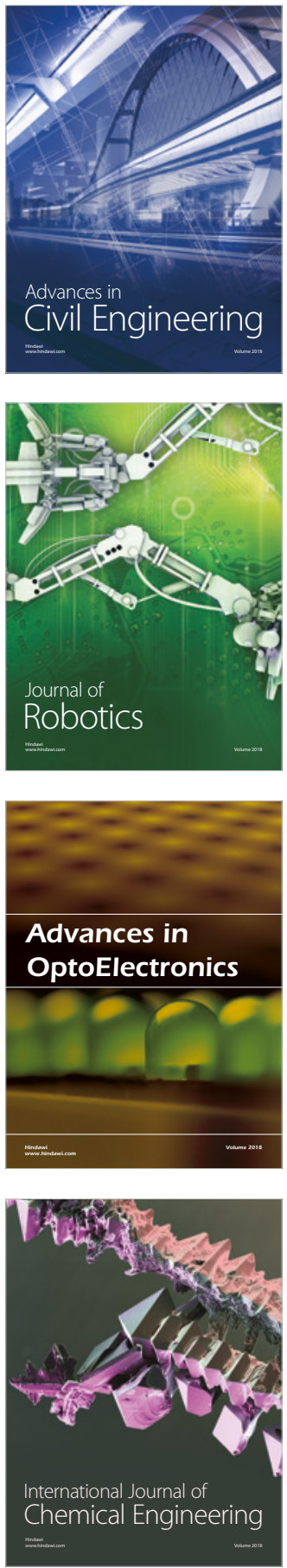

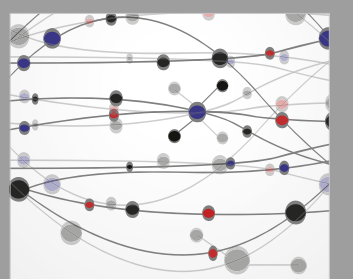

\section{Rotating \\ Machinery}

The Scientific World Journal

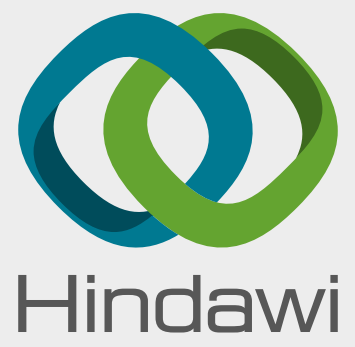

Submit your manuscripts at

www.hindawi.com
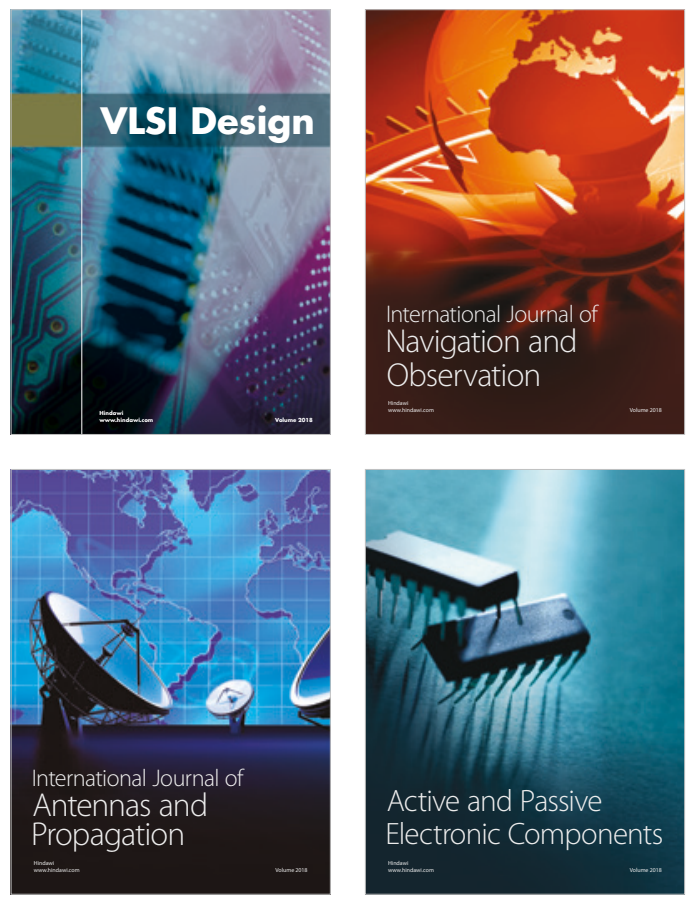
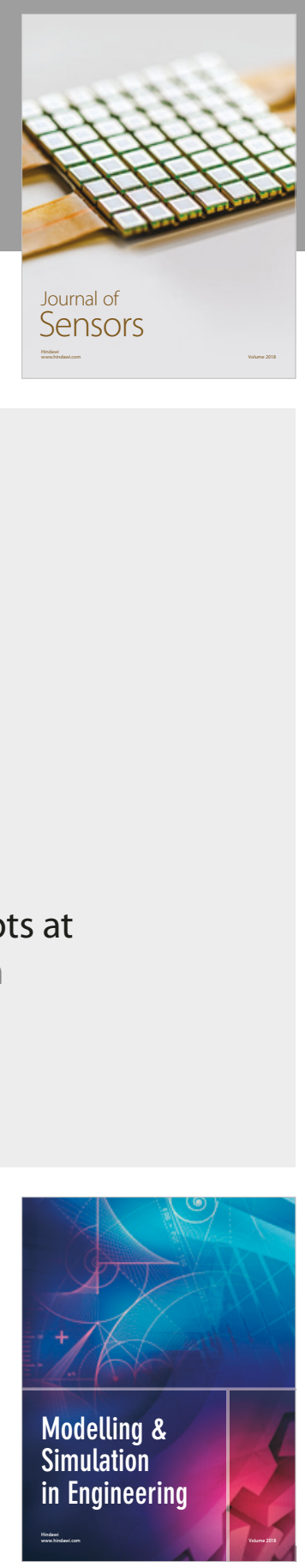

\section{Advances \\ Multimedia}
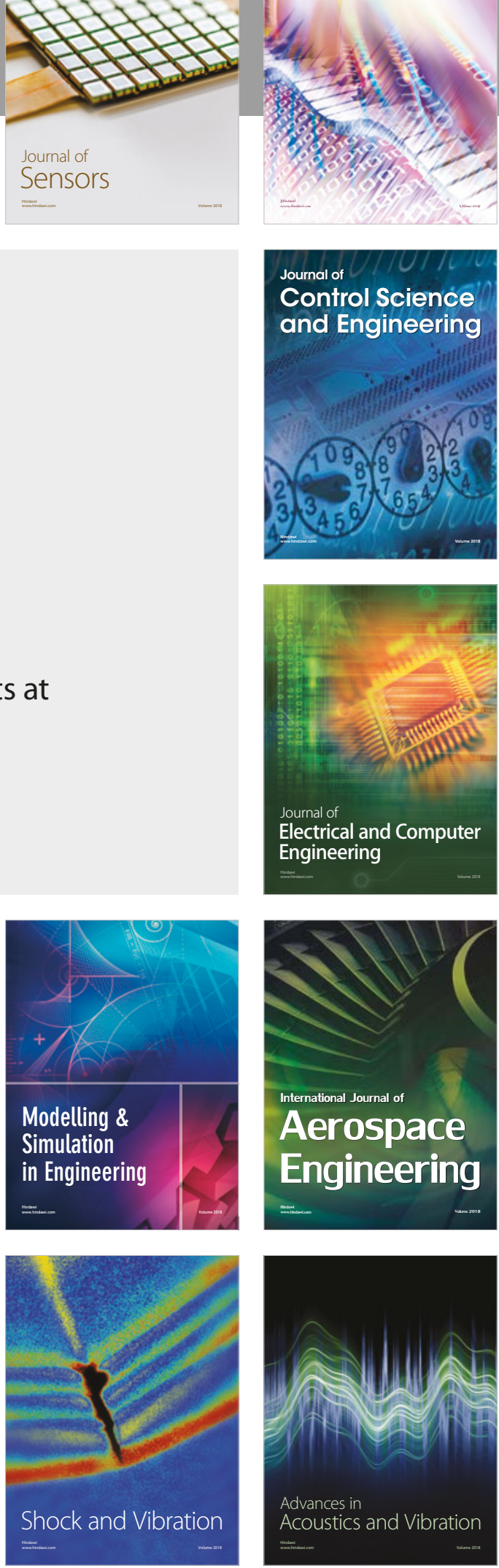